\title{
Entropy Growth and Formation of Stationary Entanglement due to Intrinsic Noise in the Two-Mode JC Model
}

\author{
H. A. Hessian \\ Mathematics Department, Faculty of Science, Assiut University, Assiut, Egypt
}

Received: 27 Aug. 2012, Revised: 21 Nov. 2012, Accepted: 23 Nov. 2012

Published online: 1 Apr. 2013

\begin{abstract}
We investigate the dynamics of the two-mode Jaynes-Cummings model with generalized nonlinear coupling including intrinsic noise as described by the Milburn equation. Based on a general analytic solution of the corresponding master equation, we calculate the growth of entropy in the system at the special case of two Raman-coupled long lived atomic states as well as the partial entropies of the atom and field subsystem. We see that the system is very sensitive to phase noise, if both fields are initially in a coherent state. The dynamics then rapidly leads to suppression of quantum revivals and a steady entanglement between the fields and the atom. This hints for serious limitations for the use of Raman induced transitions in quantum information processing setups. For an alternative entanglement measure we calculate the negativity of the eigenvalues of the partially transposed density matrix and compare it to the difference of the total entropy to the sum of field and atom partial entropies.
\end{abstract}

Keywords: Entropy, Entanglement, Decoherence

\section{Introduction}

Entanglement is one of the key properties distinguishing quantum theory from classical descriptions of the world and arises in almost any coherent interaction between distinct subsystems. Naturally it has been a subject of study for decades. Recently there has been a very intense renewed interest in the fundamental properties of entanglement as it plays a central role in quantum information processing. Examples are quantum teleportation [1], quantum dense coding [2], quantum secret sharing [3] or quantum computation[4]. New measures on quantifying entangled states has been developed by several authors [5-7]. Beyond this still many problems to fully understand the concept of entanglement rather than to knowing its mathematical definition remain.

Entanglement is ubitiquous in multipartite systems. An important practical aspect is, however, the study of systems that can be used to generate entanglement in a controlled way. The prototype system in quantum optics to generate and observe entanglement consists of a single field mode coupled to a single atom, i.e. the Jaynes-Cummings (JC) model [8-11], where the unperturbed time evolution implies a continuous generation and annihilation of entanglement. As is well known this can be easily monitored quantitatively by the size of the partial entropies of the field $S_{f}$ or the atom $S_{a}$ [7]. One finds that the higher the entropies $\left(S_{f}, S_{a}\right)$, the greater the entanglement. Starting from an initial atom-field product state one can find perfectly entangled states between field and atom at certain later times even for initial coherent states with large photon number [8-10] which almost vanishes again at the revival time.

It is important to note that the time evolution of the field (atomic) entropy reflects the time evolution of the degree of entanglement only, if one deals with a pure state of the system with zero total entropy $S=0$. Any environmental coupling introduces entropy growth in the observed subsystem. Naturally some damping is always present experimentally and it is conventionally modelled by weak coupling to an external thermal reservoir including energy exchange. In such a dissipative quantum system the interaction leads to a slow thermalization of the system and a decay of any entanglement. As is well known damping has a very severe effect on quantum coherences of the system involving superposition states of

\footnotetext{
${ }^{*}$ Corresponding author e-mail: ammar $67 @$ yahoo.com
} 
well distinguishable components [12-14]. These damp out on a much faster time scale. From this one can expect a strong sensitivity of entanglement generation on noise even on a shorter time scale. Several authors have thus studied the JC model with damping by the use of analytic approximations [15-17] and numerical calculations [18-21]. Recently it has been demonstrated that entanglement is generated in a JC model for a pure state of one of its subsystems and the other in a mixed state [22].

In a practical implementation to generate entanglement between two subsystems one tries to reduce the environmental energy exchange and coupling with the rest of the world as much as possible. Any generated entanglement between the environment and the considered systems is a potential information leak and degrades the quality of the generated entanglement. Unfortunately besides energy input-output, there are other kinds of environmental influences, which do not involve energy exchange, but rather induce fluctuations of the system energy levels and coupling phases. A prototype dynamics to study the dynamics of this so called phase damping has been proposed by Milburn [23]. Here the system-environment interaction Hamiltonian commutes with that of the system. Dynamically only the phase of the quantum state is changed in the interaction. This implies that off diagonal elements of the density matrix in the energy basis decay at a given rate. Apart from providing a consistent frame to study such couplings, including effective phase damping terms often well describes unaccounted decay of coherences in experimental setups as found e.g in a single mode micro maser[24]. In addition, it has been shown recently, that phase damping seriously reduces the fidelity of the received qubit in quantum communication[25]. Of course phase damping has been investigated not only in the JC model but also in other more complex quantum systems as quantum computers transport phenomena in mesoscopic system [26].

Here we study the influence of phase noise on the growth of the system entropy and the formation of entanglement at the analytically solvable example of multi-quanta two-mode JC model. Nonlinear couplings between two modes are typical ingredients to build quantum gate operations. As the simplest nontrivial example we will then consider a Raman-type coupling between two long lived atomic states by the two modes, which we like to manipulated in a well defined way. Such a Raman coupling can be used to generated controlled entanglement between the two modes. However, at the same time during this process also entanglement between the modes and the atom is generated, which is often neglected but degrades the entanglement between the two fields. This complicates e.g. the achievement of perfect entanglement of the two modes. By a suitable choice of coupling strengths and time this problem can be circumvented in principle. In the following we will investigate, how the addition of phase noise generates even more stringent conditions to reach this goal. In particular we consider the case, when one uses weak coherent fields instead of photon number eigenstates to perform the task.

As said before due to the growth of the total entropy, calculating simply the partial atomic entropy does not provide for a good quantitative measure of entanglement [6]. Fortunately a number of alternative possibilities to get a measure of entanglement have been developed recently [6], which can be very well used in our case. In particular we will use the negativity of the eigenvalues of the partial transpose of the density matrix as introduced by Vidal [27] recently, which provides for a convenient, computable measure of entanglement. As an extra point in our work this allows to compare the two entanglement measures quantitatively via the study of the sensitivity of entanglement formation on the amount of phase noise.

This paper is organized as follows: In section 2, we present the master equation to describe the phase damping of a quantum system under the Markovian approximation. In section 3 , we obtain an exact solution of the multi-quanta two-mode JC model with phase damping and give the explicit expression of this solution in the two-dimensional basis of the particle. Section 4 is devoted to an investigation of the sensitivity of the particle-field entanglement generation an entropy growth to the amount of phase noise and also we compare different measures of entanglement.

\section{Master equation describing phase damping}

We consider a system described by the Hamiltonian $H$ and interacting with the heat-bath environment (the reservoir) which consists of an infinite set of harmonic oscillators. we assume that the system interacting with the environment can be described by the total Hamiltonian

$$
\begin{aligned}
\hat{H}_{T}=\hat{H}+ & \sum_{i}\left(\frac{p_{i}^{2}}{2 m_{i}}+\frac{1}{2} m_{i} \omega_{i}^{2} x_{i}^{2}\right) \\
& +\hbar \hat{H} \sum_{i} C_{i} x_{i}+\hbar^{2} \hat{H}^{2} \sum_{i} \frac{\left|C_{i}^{2}\right|}{2 m_{i} \omega_{i}^{2}}
\end{aligned}
$$

where the second term is the Hamiltonian of the reservoir, the third represents the interaction between the system and the reservoir with the coupling constant $\hbar C_{i}$, and the last is the renormalization term [28]. Obviously, the system Hamiltonian $\hat{H}$ commutes with the interaction Hamiltonian in Eq.(1). The form of coupling between the system and the reservoir which we adopt here is also of importance in the back-action-evading and quantum-non demolition schemes [29]. From Louisell's approach [30], under the Markovian approximation we can obtain the following master equation describing the phase damping in the interaction picture:

$$
\frac{d}{d t} \hat{\rho}(t)=\frac{1}{i \hbar}[\hat{H}, \hat{\rho}(t)]-\gamma[\hat{H},[\hat{H}, \hat{\rho}(t)]]
$$


where $\gamma$ is an effective constant depending on the temperature and spectral density of the reservoir [31]. Obviously, the master equation (2) has the same form as the Milburn's equation [31] under the diffusion approximation.

\section{Analytic solution of the master equation}

In this section, we shall consider a Hamiltonian model that consists of two modes interacting with a three-level particle (atom or trapped ion) via Raman transition. We consider the nondegenerate case in which pairs of photons with two different frequencies are created or annihilated. The atomic levels have identical parities such that each dipole is coupled with different modes of the field and to the set of intermediate states. If we assume that the intermediate states do not admit dipole transitions between themselves and the interaction field modes are far off-resonance from those intermediate states, then the particle can be seen as an effective two-level system by means of adiabatic elimination of the intermediate state $[32,33]$. The Hamiltonian for the system, in the rotating wave approximation, is written as:

$$
\begin{aligned}
\hat{H}= & \frac{\omega_{\circ}}{2} \hat{\sigma}_{z}+\sum_{j=1}^{2} \omega_{j} \hat{a}_{j}^{\dagger} \hat{a}_{j}+\lambda\left(\hat{a}_{1}^{\dagger k_{1}} \hat{a}_{2}^{k_{2}} \hat{\sigma}_{-}+\hat{a}_{1}^{k_{1}} \hat{a}_{2}^{\dagger k_{2}} \hat{\sigma}_{+}\right) \\
= & \omega_{1}\left[\hat{n}_{1}+\frac{k_{1}}{2}\left(I+\hat{\sigma}_{z}\right)\right]+\omega_{2}\left[\hat{n}_{2}+\frac{k_{2}}{2}\left(I-\hat{\sigma}_{z}\right)\right] \\
& -\frac{1}{2}\left(k_{1} \omega_{1}+k_{2} \omega_{2}\right) I+\frac{\Delta}{2} \hat{\sigma}_{z}+\lambda\left(\hat{a}_{1}^{\dagger k_{1}} \hat{a}_{2}^{k_{2}} \hat{\sigma}_{-}\right. \\
& \left.+\hat{a}_{1}^{k_{1}} \hat{a}_{2}^{\dagger k_{2}} \hat{\sigma}_{+}\right),
\end{aligned}
$$

where the detuning parameter $\Delta=\omega_{\circ}-k_{1} \omega_{1}+k_{2} \omega_{2}, \hat{a}_{j}$ $\left(\hat{a}_{j}^{\dagger}\right)$ and $\hat{n}_{j}=\hat{a}_{j}^{\dagger} \hat{a}_{j}$ are the annihilation (creation) and number operators for the $j t h$ mode, $\lambda$ is the particle-field coupling constant, $\omega_{1}$ and $\omega_{2}$ are the field frequencies for the two modes, $\omega_{\circ}$ is the transition frequency of the particle (atom or trapped ion), $\hat{\sigma}_{z}$ is the population inversion operator, while $k_{1}$ and $k_{2}$ are the multi-quanta parameters and $\hat{\sigma}_{ \pm}$are the 'spin flip' operators which satisfy the relation $\left[\hat{\sigma}_{+}, \hat{\sigma}_{-}\right]=\hat{\sigma}_{z}$ and $\left[\hat{\sigma}_{z}, \hat{\sigma}_{ \pm}\right]= \pm 2 \hat{\sigma}_{ \pm}$.

Now, we look for the exact solution for the density operator $\hat{\rho}(t)$ of the master equation (2) taking into account the Hamiltonian (3).

For convenience, we introduce three auxiliary superoperators [34-36] $\hat{J}, \hat{S}$ and $\hat{L}$ defined by

$$
\begin{gathered}
\exp (\hat{J} \tau) \hat{\rho}(t)=\sum_{k=0}^{\infty} \frac{(2 \tau \gamma)^{k}}{k !} \hat{H}^{k} \hat{\rho}(t) \hat{H}^{k}, \\
\exp (\hat{S} \tau) \hat{\rho}(t)=\exp (-i \hat{H} \tau) \hat{\rho}(t) \exp (i \hat{H} \tau), \\
\exp (\hat{L} \tau) \hat{\rho}(t)=\exp \left[-\gamma \tau \hat{H}^{2}\right] \hat{\rho}(t) \exp \left[-\gamma \tau \hat{H}^{2}\right],
\end{gathered}
$$

It is straightforward to obtain the formal solution of the master equation (Eq. 2) as follows:

$$
\hat{\rho}(t)=\exp (\hat{J} t) \exp (\hat{S} t) \exp (\hat{L} t) \hat{\rho}(0)
$$

where $\hat{\rho}(0)$ is the density operator of the initial particle-field system. We assume that the initial two modes of the field inside the cavity are in a coherent state and the particle in its excited state $\mid e>$ or ground state $\mid g>$, so that:

$$
\hat{\rho}^{e}(0)=\left|\alpha_{1}, \alpha_{2}><\alpha_{1}, \alpha_{2}\right| \otimes|e><e|,
$$

or

$$
\hat{\rho}^{g}(0)=\left|\alpha_{1}, \alpha_{2}><\alpha_{1}, \alpha_{2}\right| \otimes|g><g|,
$$

with $\left|\alpha_{1}, \alpha_{2}>=\right| \alpha_{1}>\otimes \mid \alpha_{2}>$ defined by

$$
\left|\alpha_{1}, \alpha_{2}>=\sum_{n_{1}, n_{2}=0}^{\infty} q_{n_{1}} q_{n_{2}}\right| n_{1}>\otimes \mid n_{2}>
$$

where $q_{n_{j}}=e^{-\alpha_{j}^{2} / 2} \frac{\alpha_{j}^{n_{j}}}{\sqrt{n_{j} !}},(j=1,2)$.

Following essentially the same procedures as in Refs. [34-36], we arrive at the explicit expression of the exact solution of the master equation (2) for multi-quanta twomode JC model in the following form:

$$
\begin{aligned}
\hat{\rho}(t)= & \sum_{k=0}^{\infty} \frac{(2 \gamma t)^{k}}{k !}\left[\hat{M}_{e e}^{(k)}(t)|e><e|+\hat{M}_{e g}^{(k)}(t)|e><g|\right. \\
& \left.+\hat{M}_{g e}^{(k)}(t)|g><e|+\hat{M}_{g g}^{(k)}(t)|g><g|\right], \quad \text { (11) }
\end{aligned}
$$

where

$\hat{M}_{e e}^{(k)}(t)=\hat{A} \hat{\Psi}_{11}(t) \hat{A}+\hat{A} \hat{\Psi}_{12}(t) \hat{C}+\hat{B} \hat{\Psi}_{21}(t) \hat{A}+\hat{B} \Psi_{22}(t) \hat{C}$

$\hat{M}_{g g}^{(k)}(t)=\hat{C} \hat{\Psi}_{11}(t) \hat{B}+\hat{C} \hat{\Psi}_{12}(t) \hat{D}+\hat{D} \hat{\Psi}_{21}(t) \hat{B}+\hat{D} \hat{\Psi}_{22}(t) \hat{D}$

and

$$
\begin{aligned}
\hat{M}_{g e}^{(k)}(t)=\left[\hat{M}_{e g}^{(k)}(t)\right]^{\dagger}= & \hat{C} \hat{\Psi}_{11}(t) \hat{A}+\hat{C} \hat{\Psi}_{12}(t) \hat{C} \\
& +\hat{D} \hat{\Psi}_{21}(t) \hat{A}+\hat{D} \hat{\Psi}_{22}(t) \hat{C}
\end{aligned}
$$

with

$$
\begin{aligned}
& \hat{A}=\left[\hat{f}_{1}^{(k)}+\left(\frac{\Delta}{2 \lambda}\right) \hat{g}_{1}^{(k)^{\prime}}\right], \quad \hat{B}=\hat{a}_{1}^{k_{1}} \hat{a}_{2}^{\dagger k_{2}} \hat{g}_{2}^{(k)^{\prime}}, \\
& \hat{C}=\hat{g}_{2}^{(k)^{\prime}} \hat{a}_{1}^{\dagger k_{1}} \hat{a}_{2}^{k_{2}}, \quad \hat{D}=\left[\hat{f}_{2}^{(k)}-\left(\frac{\Delta}{2 \lambda}\right) \hat{g}_{2}^{(k)^{\prime}}\right],
\end{aligned}
$$

where

$$
\hat{\Psi}_{i j}(t)=\left|\hat{\Psi}_{i}(t)><\hat{\Psi}_{j}(t)\right| \quad(i, j=1,2),
$$

If the particle starts from its excited state, i.e., with the initial condition (8), then

$$
\left|\hat{\Psi}_{1}(t)>=\left[\hat{R}_{1}(t)-\left(\frac{\Delta}{2 \lambda}\right) \frac{\hat{V}_{1}(t)}{\hat{F}_{1}}\right]\right| \hat{\Psi}(t)>,
$$




$$
\begin{aligned}
\left|\hat{\Psi}_{2}(t)>=-\hat{a}_{1}^{\dagger k_{1}} \hat{a}_{2}^{k_{2}}\left[\frac{\hat{V}_{1}(t)}{\hat{F}_{1}}\right]\right| \hat{\Psi}(t)>, \\
\mid \hat{\Psi}(t)>=\exp \left[-\gamma t\left[\hat{W}_{1}^{2}+\lambda^{2} \hat{F}_{1}^{2}\right]\right] \\
\quad \times \exp \left[-i \hat{W}_{1} t\right] \mid \alpha_{1}, \alpha_{2}>.
\end{aligned}
$$

While if the particle starts from its ground state, i.e., with the condition (9), then

$$
\begin{aligned}
\left|\hat{\Psi}_{1}(t)>=-\hat{a}_{1}^{k_{1}} \hat{a}_{2}^{\dagger k_{2}}\left[\frac{\hat{V}_{2}(t)}{\hat{F}_{2}}\right]\right| \hat{\Psi}^{\prime}(t)>, \\
\left|\hat{\Psi}_{2}(t)>=\left[\hat{R}_{2}(t)+\left(\frac{\Delta}{2 \lambda}\right) \frac{\hat{V}_{2}(t)}{\hat{F}_{2}}\right]\right| \hat{\Psi}^{\prime}(t)>, \\
\mid \hat{\Psi}^{\prime}(t)>=\exp \left[-\gamma t\left[\hat{W}_{2}^{2}+\lambda^{2} \hat{F}_{2}^{2}\right]\right] \\
\times \exp \left[-i \hat{W}_{2} t\right] \mid \alpha_{1}, \alpha_{2}>
\end{aligned}
$$

and

$$
\hat{g}_{2}^{(k)^{\prime}}=\frac{\hat{g}_{2}^{(k)}}{\hat{F}_{2}}, \quad \hat{W}_{1}=w_{1}\left(n_{1}+k_{1}\right)+w_{2} n_{2}
$$

where the operators $\hat{f}_{2}^{(k)}, \hat{g}_{2}^{(k)}$ and $\hat{\Phi}_{2}( \pm)$ are defined by

$$
\begin{aligned}
& \hat{f}_{2}^{(k)}=\frac{1}{2}\left[\hat{\Phi}_{2}^{k}(+)+\hat{\Phi}_{2}^{k}(-)\right] \\
& \hat{g}_{2}^{(k)}=\frac{1}{2}\left[\hat{\Phi}_{2}^{k}(+)-\hat{\Phi}_{2}^{k}(-)\right] \quad \hat{\Phi}_{2}( \pm)=\hat{W}_{2} \pm \lambda \hat{F}_{2}, \\
& \text { with } \\
& \begin{array}{c}
\hat{R}_{2}(t)=\hat{C}_{2}(t) \hat{X}_{2}(t)+i \hat{S}_{2}(t) \hat{Y}_{2}(t) \\
\hat{V}_{2}(t)=\hat{C}_{2}(t) \hat{Y}_{2}(t)+i \hat{S}_{2}(t) \hat{X}_{2}(t) \\
\hat{C}_{2}(t)=\cos \lambda t \hat{F}_{2} \quad \hat{S}_{2}(t)=\sin \lambda t \hat{F}_{2} \\
\hat{X}_{2}(t)=\cosh \left[2 \lambda t \gamma \hat{W}_{2} \hat{F}_{2}\right] \quad \hat{Y}_{2}(t)=\sinh \left[2 \lambda t \gamma \hat{W}_{2} \hat{F}_{2}\right] \\
\hat{F}_{2}^{2}=\left(\frac{\Delta}{2 \lambda}\right)^{2}+\hat{v}_{2}^{2}, \quad \hat{v}_{2}^{2}=\frac{n_{1} !}{\left(n_{1}-k_{1}\right) !} \frac{\left(n_{2}+k_{2}\right) !}{\left(n_{2}\right) !} .
\end{array}
\end{aligned}
$$

Note that in the above equations (15-29), we have used the subscript 1 instead of $\left(n_{1}+k_{1}, n_{2}\right)$ and 2 instead of $\left(n_{1}, n_{2}+k_{2}\right)$. We can obtain the reduced density operator for the particle by taking the trace over the states of the field. Thus the reduced density particle operator becomes

$$
\hat{\rho}_{a}(t)=\operatorname{Tr}_{f} \hat{\rho}(t)=\left[\begin{array}{ll}
C_{e e}(t) & C_{e g}(t) \\
C_{g e}(t) & C_{g g}(t)
\end{array}\right]
$$

where

$$
\begin{gathered}
C_{i j}(t)=\sum_{k, n=0}^{\infty} \frac{(2 \gamma t)^{k}}{k !}<n_{1}, n_{2}\left|\hat{M}_{i j}^{(k)}(t)\right| n_{1}, n_{2}>, \\
(i, j=e, g),
\end{gathered}
$$

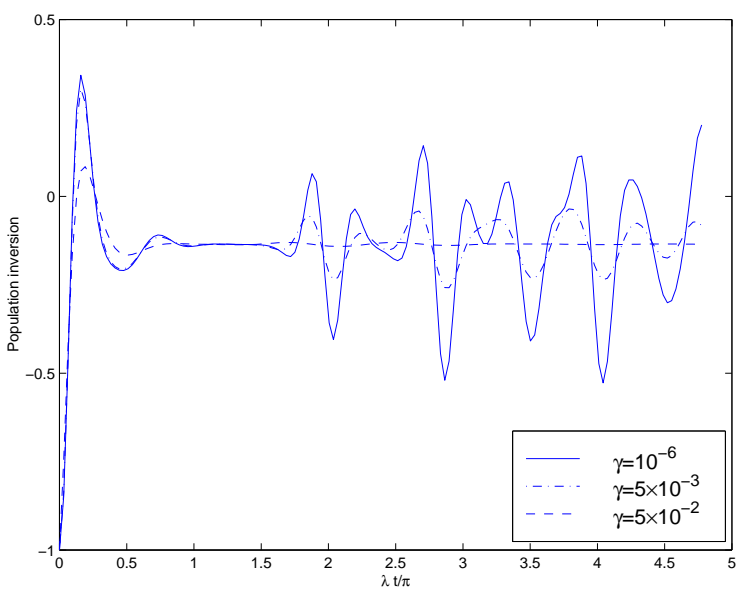

Fig. 1: The population inversion for the particle initially prepared in the ground state $\mid g>$, for various values of the parameter $\gamma$.

while the reduced density operator for the field is given by

$$
\hat{\rho}_{f}(t)=\operatorname{Tr}_{a} \hat{\rho}(t)=\sum_{k=0}^{\infty} \frac{(2 \gamma t)^{k}}{k !}\left[\hat{M}_{e e}^{(k)}(t)+\hat{M}_{g g}^{(k)}(t)\right] .
$$

Employing the density operator and the reduced density operators for the particle or the field are given by equations (11), (30), and (32), we investigate the properties of the evolution of the degree of entanglement.

\section{Degree of entanglement}

Let us first have a look on the typical time evolution of the system by calculating the population inversion as function of time for the atom starting in the lower state and the fields being in weak coherent states. Here (Fig.1 -solid curve) one finds the well known Rabi oscillations of the atomic population which damp out fast due to the uncertainty in the photon number but show a revival after some longer time. These revivals prove to be very sensitive to even a small amount of phase noise (dot-dashed and dashed curves) and the system reaches a steady state, where both levels are similarly populated.

The field of quantum information and computing is based on the manipulation of quantum coherent states [37]. Existing device of quantum optics has been proposed as experimental implementation and is employed to realize quantum computers. This in fact encouraged us to extend the previous work [38] to the case of two modes interacting with a two-level atom, to discuss the effect of the entropy and entanglement where the multiphoton processes are involved. Therefore, our target in the present paper is to concentrate on the two-mode case rather than the case of one-mode, which has been previously considered. 


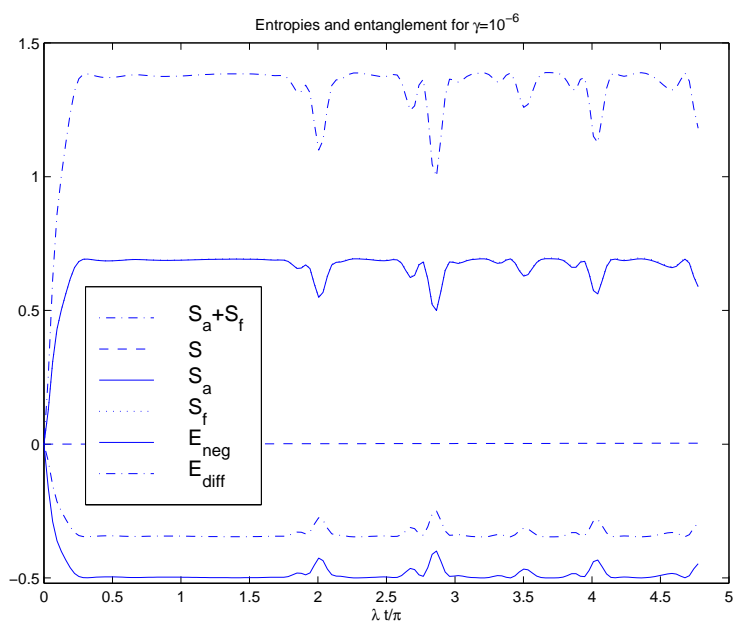

Fig. 2: Partial entropy $S_{a}$ for the atom (upper solid curve), $S_{f}$ the field (dotted curve) as well as the total entropy $S$ (dashed curve) and $S_{a}+S_{f}$ (dot-dashed curve) as a function of the scaled time $\lambda t$ of the particle initially prepared in the excited state $\mid e>$ and the field initially prepared in a coherent state $\left|\alpha_{1}, \alpha_{2}\right\rangle$ $\left(\bar{n}_{1}=2, \bar{n}_{2}=3\right.$ ), for small phase noise $\gamma=10^{-6}$. The lower solid curve shows the sum of the negative eigenvalues $E_{\text {neg }}$ of the partially transposed density matrix $\rho^{T_{A}}$ in comparison to the entropy difference $E_{\text {diff }}$ (lower dot-dashed curve).

In the previous work[38], we studied how intrinsic decoherence leads to growing entropy and a strong degradation of the maximal generated entanglement in the single mode JC model. Also, we observed an increase in the values of the entropies $S$ and $S_{f}$, while $S_{a}$ converges to 0.5 as t develops.

In this article, we investigate the dynamics of the two-mode JC model with generalized nonlinear coupling including intrinsic noise as described by the Milburn equation. Based on a general analytic solution of the corresponding master equation, we calculate the growth of entropy in the system at the special case of two Raman-coupled long lived atomic states as well as the partial entropies of the atom and field subsystem. We see that the system is very sensitive to phase noise, if both fields are initially in a coherent state. For an alternative entanglement measure we calculate the negativity of the eigenvalues of the partially transposed density matrix and compare it to the difference of the total entropy to the sum of field and atom partial entropies. As we see in the next sec., we observe increase in the values of the entropies $S$ and $S_{f}$, while $S_{a}$ converges to 0.7 after short time. In this case the field subsystem has higher sub-entropy for increased $\gamma$ which can be taken as a stronger degree of correlation than for smaller value of $\gamma$. On the other hand the atomic subsystem shows slight decrease in its sub-entropy as $\gamma$ increases. Also, one notes that the entanglement for the field subsystem grows faster for the initial ground state of the atom than the initial excited state.

Let us now investigate the influence of phase damping on the evolution of the fields, the atomic and the total entropy for two-mode JC model at the hand of numerical examples.

It is now in interesting question whether and how much the atom and the fields get entangled in this process[6]. Here we will compare two methods to quantify this. One the one hand we get a qualitative estimate of the entanglement by calculating the relative size of the total entropy of the system and the sum of the entropies in each subsystem. Alternatively we use the recently proposed measure involving the sum of negative eigenvalues $E_{n e g}$ of the partially transposed density matrix $\rho^{T_{A}}$ as a proven quantitative measure [27].

Let us now briefly repeat some of key underlying definitions. The entropy $S$ of a quantum-mechanical system described by the density operator $\hat{\rho}$ is defined as follows:

$$
S=-\operatorname{Tr}\{\hat{\rho} \ln \hat{\rho}\}
$$

where we have set the Boltzmann constant $K$ equal to unity. If $\hat{\rho}$ describes a pure state, then $S=0$, and if $\hat{\rho}$ describes a mixed state, then $S \neq 0$. Entropies of the atomic and field sub-systems are defined by the corresponding reduced density operators:

$$
S_{a(f)}=-\operatorname{Tr}_{a(f)}\left\{\hat{\rho}_{a(f)} \ln \rho_{a(f)}\right\} .
$$

For the entropy of a general two-component system one has the Araki-Lieb theorem [39]: $\left|S_{a}-S_{f}\right| \leq S \leq S_{a}+S_{f}$. One immediate consequence of this inequality is that if the total system is in a pure state, then the component systems have equal entropies $[8,10]$. If these are nonzero, it clearly proves entanglement in this case as for any product density operator the total entropy is the sum of the subsystem entropies. For a general state one has entanglement, if the subsystem entropies have a sum bigger than the total entropy well established.

We shall consider the entropy difference $E_{\text {diff }}$ defined by $E_{\text {diff }}=\frac{1}{4}\left(S-S_{a}-S_{f}\right)$. Negative values of this quantity means a degree of entanglement. We will compare this difference with the sum of negative eigenvalues of the partially transposed density matrix as a recently proposed quantitative entanglement measure [27]. The negative eigenvalues of the partially transposed matrix $\rho^{T_{A}}$ of the density $\rho$. This partial transpose takes the form of a direct sum, such that the spectrum of $\rho^{T_{A}}$ can be evaluated from the spectrum of each block. The negative eigenvalues of this spectrum are then summed to give the value $E_{n e g}$. However, the question of quantifying the degree of entanglement for general mixed states is still under discussion [22].

\section{Numerical results and discussion}

We display the evolution of the entropies when the atom initially in the excited state as functions of the scaled time 


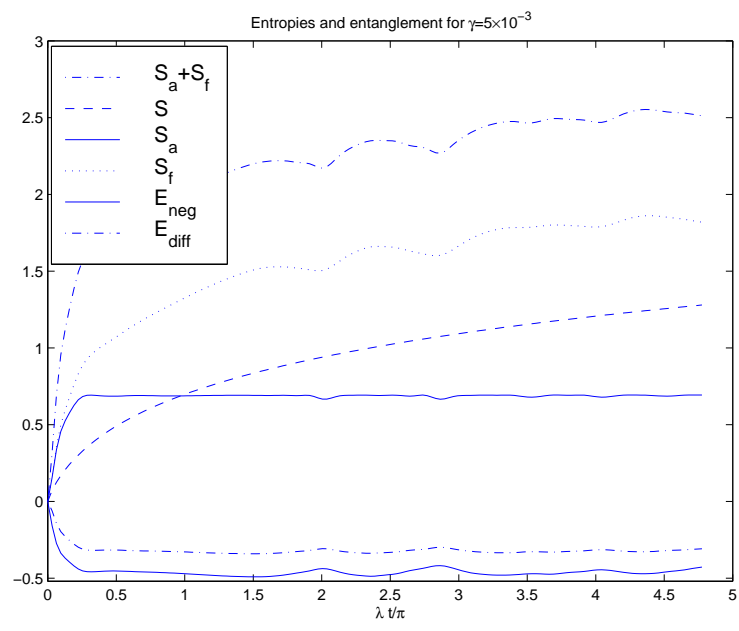

Fig. 3: Same as Fig. 2 for the phase noise $\gamma=5 \times 10^{-3}$.

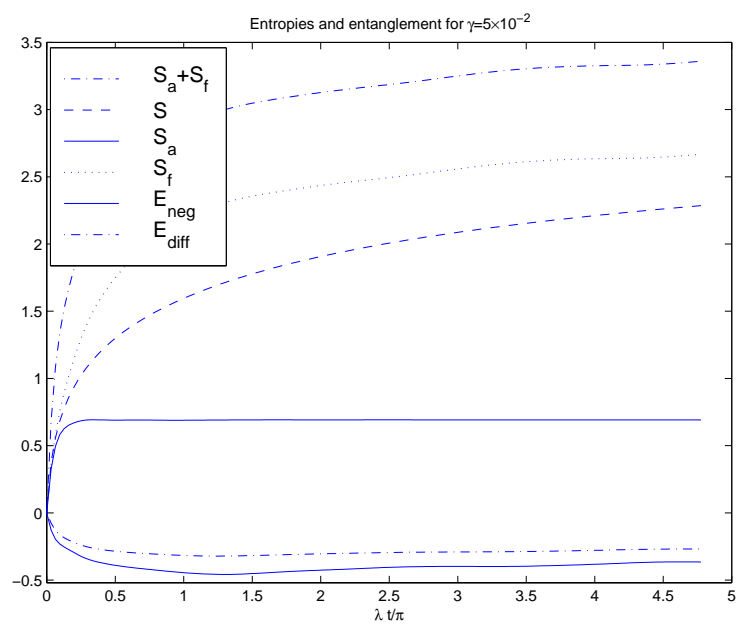

Fig. 4: Same as Fig. 2 for the phase noise $\gamma=5 \times 10^{-2}$.

$\lambda t$ for various values of the phase damping parameter $\gamma$. Here we assume a coherent field state with fixed initial mean numbers of quanta of $\bar{n}_{1}$ and $\bar{n}_{2}\left(\bar{n}_{1}=2, \bar{n}_{2}=3\right)$ for two-quanta $\left(k_{1}=k_{2}=1\right)$ in the exact resonance i.e., the detuning parameter $\left(\frac{\Delta}{2 \lambda}\right)=0$.

In Figures 2- 4 (for the initial excited state of the atom) and 5-7 (for the initial grounded state of the atom ), we display the entropy for the atom $S_{a}$, field entropy $S_{f}$ as well as the total entropy $S$ of the system, sum of the partial entropies, entropy difference $E_{\text {diff }}$ and sum of the negative eigenvalues of the partially transposed density matrix $E_{\text {neg }}$ for three small values of the noise parameter $\gamma:$ (a) $10^{-6}$, (b) $5 \times 10^{-3}$ and (c) $5 \times 10^{-2}$ for both cases the atom initially in excited state and grounded state, respectively.

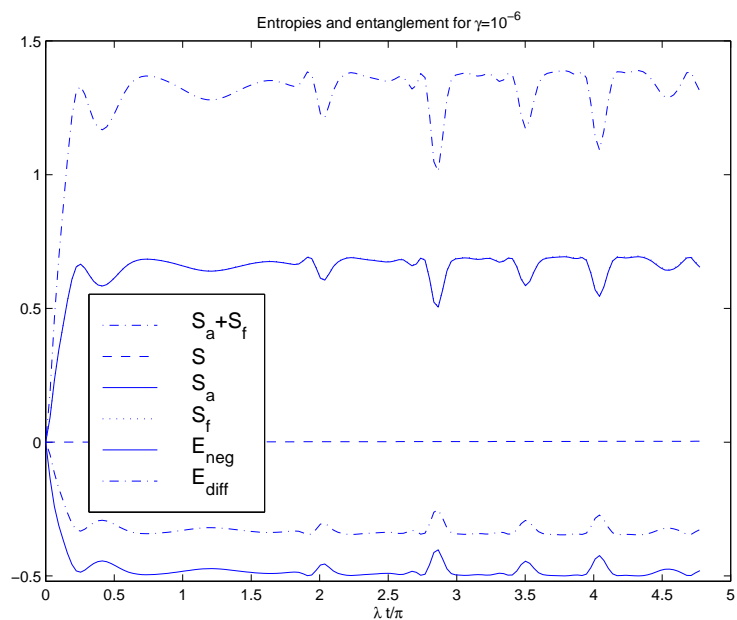

Fig. 5: Partial entropy $S_{a}$ for the atom (upper solid curve), $S_{f}$ the field (dotted curve) as well as the total entropy $S$ (dashed curve) and $S_{a}+S_{f}$ (dot-dashed curve) as a function of the scaled time $\lambda t$ of the particle initially prepared in the ground state $|g\rangle$ and the field initially prepared in a coherent state $\left|\alpha_{1}, \alpha_{2}\right\rangle$ $\left(\bar{n}_{1}=2, \bar{n}_{2}=3\right)$, for small phase noise $\gamma=10^{-6}$. The lower solid curve shows the sum of the negative eigenvalues $E_{\text {neg }}$ of the partially transposed density matrix $\rho^{T_{A}}$ in comparison to the entropy difference $E_{\text {diff }}$ (lower dot-dashed curve).

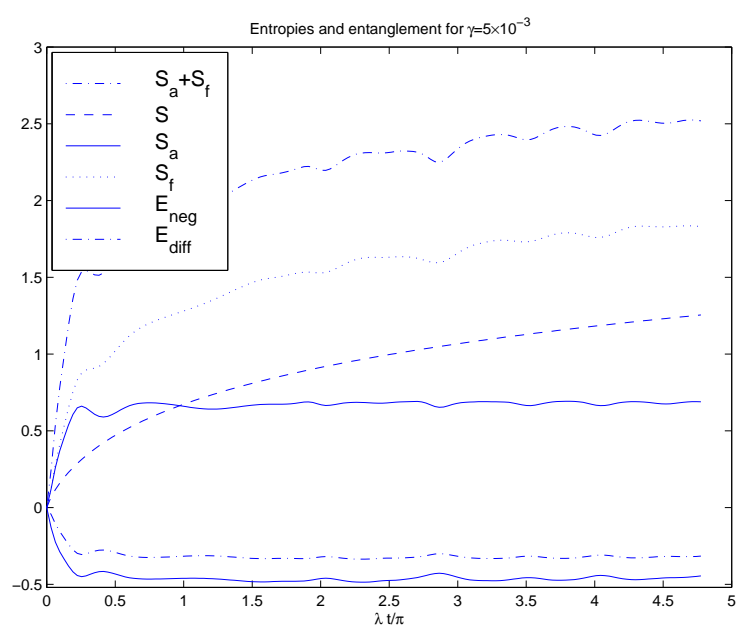

Fig. 6: Same as Fig. 5 for the phase noise $\gamma=5 \times 10^{-3}$.

In Figures 2 and 5, we show the temporal evolution of the partial atomic and field entropies $\left(S_{a}, S_{f}\right)$ and the total entropy $S$ for very weak noise in both cases. As entropy measures we plot the sum of the negative eigenvalues of the partially transposed density matrix $E_{n e g}$ and the entropy difference $E_{\text {diff }}$.

From these figures the entropies $\left(S_{f}\right.$ (upper dotted curve), $S_{a}$ (upper solid curve)), we observe that both $S_{f}$ 


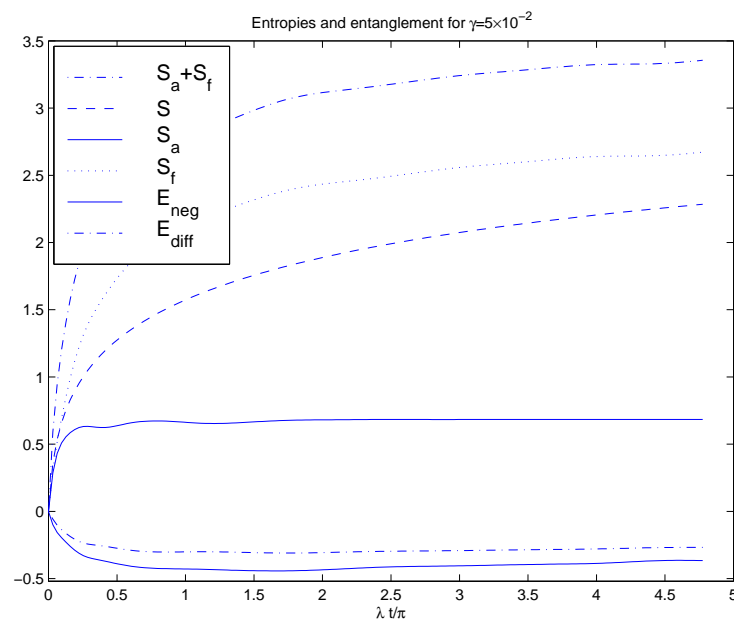

Fig. 7: Same as Fig. 5 for the phase noise $\gamma=5 \times 10^{-2}$.

and $S_{a}$ are equal and the total entropy $S=0$ (see Figures 2 and 5). They start from the value $S_{a}=S_{f}=0$, which means disentanglement then both of these subsystem show entanglement. The degree of entanglement fluctuations when time progresses as can be demonstrated in these figures.

By increasing of the phase noise parameter $\gamma$, we note that the total entropy increases monotonically, and no longer equals to zero. The partial entropies for the atom and the field are no longer equal, and hence can not be used as measure for entanglement. It is remarked that the field attains higher entropy than the atom. However, the atomic entropy is less than the total entropy for this value, while the field entropy is higher than the total entropy. We observe increase in the values of the entropies $\mathrm{S}$ and $S_{f}$, while $S_{a}$ converges to 0.7 as t develops (see Figures 3,4 , 6 , and 7). In this case the field subsystem has higher sub-entropy for increased $\gamma$ which can be taken as a stronger degree of correlation than for smaller value of $\gamma$. On the other hand the atomic subsystem shows slight decrease in its sub-entropy as $\gamma$ increases.

This sensitivity becomes even more clearly visible, when we show the total entropy of the system $S$ and compare it to the sum of partial entropies $S_{a}+S_{f}$ and the sum of negative eigenvalues $E_{n e g}$ of $\rho^{T_{A}}$. This is shown in these figures for both cases.

Also from these figures, we show the time evolution of the entropy difference $E_{\text {diff }}$ (lower dotted-dashed curve) and sum of the negative eigenvalues of the partially transposed density matrix $E_{\text {neg }}$ (lower solid curve) when the atom in both cases (excited and ground state). As we see from these figures, we get a degree of entanglement, which is well reflected as a very good measure.

Here again we note that $E_{\text {neg }}$ and $E_{\text {diff }}$ show the same characters for the maxima and minima for small $\gamma$. But as $\gamma$ increases to $5 \times 10^{-2}$ it is observed that $E_{\text {diff }}$ shows more fluctuations than $E_{n e g}$. However, in both cases as $\gamma$ increases neither $E_{\text {neg }}$ nor $E_{\text {diff }}$ reaches the value zero for any time $t>0$ which means that disentanglement does not occur at any time later.

To visualize the influence of phase damping on the evolution of the entropies, we set different values of the parameter $\gamma\left(5 \times 10^{-3}, 5 \times 10^{-2}\right)$ (see Figures $(3,4),(6$, and 7)) and all the parameter are the same as in Figures 2 and 5 .

These figures show that with the increasing of the parameter $\gamma$, we observe rapid deterioration of the fluctuations of the field and atom entropy. This shows a faster approach to the steady state due to phase damping in the nonlinear coupling case.

\section{Conclusions}

In this paper we calculated the influence of phase damping on the evolution of the field, atom and total entropy for two-mode JC model. We find a large amount of generated entanglement in the steady state limit. It shows a strong sensitivity on the amount of phase noise described by the parameter $\gamma$. Numerical studies show that the entanglement for the atomic subsystem with the atom starting in its excited state exhibits faster approach steady state than when the atom starts in its ground state . On the other hand one notes that the entanglement for the field subsystem grows faster for the initial ground state of the atom than the initial excited state. This could imply strong restrictions on the time scale of quantum gate operations, if one has fluctuating energy levels, phase noise or not well defined interaction times. We find that the relative size of partial system and total entropies provides for qualitatively correct estimate of entanglement but shows only limited quantitative agreement with the alternative entanglement measure using the negative eigenvalues of the partially transposed density matrix.

\section{Acknowledgment}

The author would like to thank the Prof. Dr. Helmut Ritsch, Institute of Theoretical Physics, Innsbruck University, Innsbruck, Austria, for the hospitality and financial support under the system of associateship, where this work is done.

\section{References}

[1] C. H. Bennett, G. Brassard, C. Crépeau, R. Jozsa, A. Peres and W. K. Wootters, "Teleporting an unknown quantum state via dual classical and Einstein-Podolsky-Rosen channels," Phys. Rev. Lett. 70, 1895-1899 (1993). 
[2] C. H. Bennett and S. Wiesner, "Communication via one- and two-particle operators on Einstein-Podolsky-Rosen states," Phys. Rev. Lett. 69, 2881-2884 (1992).

[3] M. Hillery, V. Bužek and A. Berthiaume, "Quantum secret sharing," Phys. Rev. A 59, 1829-1834 (1999).

[4] M. A. Nielsen and I. L. Chuang, Quantum Computation and Quantum Information (Cambridge: Cambridge University Press, 2000)

[5] V. Vedral, M. Plenio, M. A. Rippin and P. L. Knight, "Quantifying Entanglement," Phys. Rev. Lett. 78, 2275-2279 (1997).

[6] C. H. Bennett, D. P. Di Vincenzo, J. A. Smolin and W. K. Woottters, "Mixed-state entanglement and quantum error correction," Phys. Rev. A 54, 3824-3851 (1996); L. Henderson and V. Vedral, "Information, Relative Entropy of Entanglement, and Irreversibility," Phys. Rev. Lett. 84, 22632266 (2000).; M. Horodecki, P. Horodecki and R. Horodecki, "Limits for Entanglement Measures," Phys. Rev. Lett. 84, 2014-2017 (2000).

[7] C. H. Bennett, H. J. Bernstein, S. Popescu and B. Schumacher, "Concentrating partial entanglement by local operations," Phys. Rev. A 53, 2046-2052 (1996).

[8] S. J. D. Phoenix and P. L. Knight, "Establishment of an entangled atom-field state in the Jaynes-Cummings model," Phys. Rev. A 44, 6023-6029 (1991); "Comment on "Collapse and revival of the state vector in the Jaynes-Cummings model: An example of state preparation by a quantum apparatus", Phys. Rev. Lett. 66, 2833- (1991).

[9] V Bužek , H. Moya-Cessa, P. L. Knight and S. J. D. Phoenix,"Schrdinger-cat states in the resonant JaynesCummings model: Collapse and revival of oscillations of the photon-number distribution," Phys. Rev. A 45, 8190-8203 (1992).

[10] S. J. D. Phoenix and P. L. Knight, "Periodicity, phase, and entropy in models of two-photon resonance," J. Opt. Soc. Am. B 7 116-124 (1990).

[11] M. F. Fang and G. H. Zhou, "Influence of atomic coherence on the evolution of field entropy in multiphoton processes," Phys. Lett. A 184, 397-402 (1994).

[12] M. Brune, F. Schmidt-Kaler, A. Maali, J. Dreyer, E. Hagley, J. M. Raimond, and S. Haroche, "Quantum Rabi Oscillation: A Direct Test of Field Quantization in a Cavity "Phys. Rev. Lett. 76, 1800- (1996).

[13] J. M. Raimond, M. Brune, and S. Haroche , "Reversible Decoherence of a Mesoscopic Superposition of Field States, "Phys. Rev. Lett. 79, 1964- (1997).

[14] W. H. Zurek, Phys. Today 4436 (1991).

[15] S. M. Barnett and P. L. Knight, " Dissipation in a fundamental model of quantum optical resonance ," Phys. Rev. A 33, 2444 (1986).

[16] R. R. Puri and G. S. Agarwal, "Finite-Q cavity electrodynamics: Dynamical and statistical aspects , "Phys. Rev. A 35, 3433 (1987).

[17] H.-J. Briegel and B.-G. Englert, "Quantum optical master equations: The use of damping bases , "Phys. Rev. A 47, 3311 (1993)

[18] J. Eiselt and H. Risken, Opt. Commun. 72351 (1989); "Quasiprobability distributions for the Jaynes-Cummings model with cavity damping," Phys. Rev. A 43, 346 (1991).

[19] M. J. Werner and H. Risken, "Quasiprobability distributions for the cavity-damped Jaynes-Cummings model with an additional Kerr medium, " Phys. Rev. A 44, 4623 (1991).
[20] J. Gea-Banacloche, "Jaynes-Cummings model with quasiclassical fields: The effect of dissipation , Phys. Rev. A 47, 2221 (1993).

[21] B. G. Englert, M. Naraschewski and A. Schenzle, "Quantum-optical master equations: An interaction picture, "Phys. Rev. A 50, 2667 (1994).

[22] S. Bose, I. Fuentes-Guridi, P. L. Knight and V. Vidral, "Subsystem Purity as an Enforcer of Entanglement," Phys. Rev. Lett. 87, 050401 (2001).; "Erratum: Subsystem Purity as an Enforcer of Entanglement [Phys. Rev. Lett. 87, 050401 (2001)]," Phys. Rev. Lett. 87, 279901 (2001).; S. Scheel, J. Eiseet, P. L. Knight and M. B. Plenio, "Hot entanglement in a simple dynamical model " quant-ph/207120.

[23] D. F. Walls and G. J. Milburn, Quantum Optics (SpringerVerlag, Berlin, 1994).

[24] H.-P. Breuer, U. Dorner, and F. Petruccione, Comput. Phys. Commun. 132, 30 (2000).

[25] I. L. Chuang and Y. Yamamoto, "Creation of a persistent quantum bit using error correction," Phys. Rev. A 55114 (1997); C. Massimo Palma, K. Suominen, and A. K. Ekert, Proc. R. Soc. London, Ser. A 452567 (1996).

[26] Mesoscopic Phenomena in Solids, edited by B. L. Altschuler, P. A. Lee and R. A. Webb (Elsevier, Amsterdam, 1991).

[27] G. Vidal and R. F. Werner, "Computable measure of entanglement," Phys. Rev. A 65, 032314 (2002).

[28] A. O. Caldeira and A. J. Leggett, Ann. Phys. (N. Y.) 149374 (1983); A. J. Leggett, S. Chakravarty, A. T. Dorsey, M. P. A. Fisher, A. Garg and W. Zweger, "Dynamics of the dissipative two-state system ," Rev. Mod. Phys. 59, 1 (1987).

[29] C. Caves, K. Thorne, R. Drever, V. Sandberg and M. Zimmermann, "On the measurement of a weak classical force coupled to a quantum-mechanical oscillator. I. Issues of principle ," Rev. Mod. Phys. 52, 341 (1980).

[30] W. H. Louisell, Quantum Statistical Properties of Radiation (Wiley, New York, 1973).

[31] G. J. Milburn, "Intrinsic decoherence in quantum mechanics," Phys. Rev. A 44, 5401-5406 (1991).; "Reply to "Comment on 'Intrinsic decoherence in quantum mechanics' ", Phys. Rev. A 47, 2415-2416 (1993).

[32] P. Alsing and M. S. Zubairy, "Collapse and revivals in a two-photon absorption process ," J. Opt. Soc. Am. B 4, 177 (1987).

[33] Gao-Xiang Li and J. Peng, ”Influences of ac Stark shifts on coherent population trapping in the atom-field coupling system via Raman two-photon processes ," Phys. Rev. A 52, 465 (1995).

[34] H.A. Hessian and H. Ritsch, "Influence of phase damping on nonclassical properties of the two-mode Jaynes?Cummings model ," J. Phys. B, 35, 4619- (2002).

[35] A.-S. F. Obada, A. M. Abdel-Hafez and H. A. Hessian, "Influence of the intrinsic decoherence on nonclassical effects in the nondegenerate bimodal multiquanta Jaynes-Cummings model," J. Phys. B 31, 5085-5104 (1998).

[36] H. Moya-Cessa, V. Buzek, M. S. Kim, and P. L. Knight, "Intrinsic decoherence in the atom-field interaction," Phys. Rev. A 48, 3900-3905 (1993).

[37] A. Steane,'Quantum Computing," Rep. Prog. Phys. 61, 117173 (1998); David P. DiVincenzo, "Quantum Computation," Science 270, 255-261 (1995). 
[38] A.-S. F. Obada and H. A. Hessian, "Entanglement generation and entropy growth due to intrinsic decoherence in the JC model," J. Opt. Soc. Am. B. 21, 1535-1542 (2004).

[39] H Araki and E Lieb, "Entropy inequalities," Commun. Math. Phys. 18, 160-170 (1970). 